\title{
p54nrb, a PSF Protein Partner, Contributes to Meningitic Escherichia coli K1-Mediated Pathogenicities
}

\author{
Lina $\mathrm{He}^{1}$, Feng Chi ${ }^{1}$, Tao Bo ${ }^{1,2}$, Lin Wang ${ }^{1,3}$, Chunhua $\mathrm{Wu}^{1}$, Ambrose Jong ${ }^{1}$, Shenghe Huang ${ }^{{ }^{*}}$ \\ ${ }^{1}$ Department of Pediatrics, Saban Research Institute of Children's Hospital Los Angeles, \\ University of Southern California, Los Angeles, USA \\ ${ }^{2}$ Department of Pediatrics, Division of Neonatology, The Second Xiangya Hospital, Central South University, Changsha, China \\ ${ }^{3}$ Department of Histology and Embryology, School of Basic Medical Science, Wuhan University, Wuhan, China \\ Email: *'shhuang@hsc.usc.edu
}

Received January 14, 2012; revised February 16, 2012; accepted February 27, 2012

\begin{abstract}
IbeA is an important invasion determinant contributing to Escherichia coli $\mathrm{K} 1$ entry into brain microvascular endothelial cells (BMEC) that is a key step in the pathogenesis of E. coli meningitis. Our previous studies have shown that IbeA-induced signaling and E. coli $\mathrm{K} 1$ invasion is mediated by two IbeA-binding proteins, vimentin, which is constitutively present in the surface of human BMECs (HBMECs), and PSF, which is inducibly expressed in both mesenchymal (endothelium) and non-mesenchymal (epithelium) cells. However, it is unknown whether p54nrb, a PSF partner protein, could contribute to the pathogenesis of $E$. coli $\mathrm{K} 1$ meningitis. Here, we reported that a $54-\mathrm{kDa}$ protein was identified by copurification with PSF through IbeA-affinity chromatography as an IbeA-binding protein, which is identical to p54nrb. Both p54nrb and PSF are RNA-binding proteins and share significant sequence homology. The specific interaction between IbeA and p54nrb was confirmed by Western blot and ligand overlay assays. Recombinant p54nrb blocked E. coli K1 invasion of human BMEC very effectively. Overexpressed p54nrb as a GFP fusion protein in the transfected 293T cells significantly enhanced E. coli K1 invasion. Furthermore, higher levels of surface p54nrb in the transfected 293T cells were detected by flow cytometry. These results suggest that the IbeA invasion protein of E. coli K1 interacts with p54nrb for bacterial invasion of human cells.
\end{abstract}

Keywords: Meningitis; Escherichia coli; BMEC; IbeA; p54nrb; Invasion Protein; Receptor; Protein Interaction

\section{Introduction}

Despite the availability of effective bactericidal antibiotics over the last sixty years neonatal bacterial meningitis remains an important cause of high mortality and morbidity [1-4]. Escherichia coli is the most common cause of neonatal meningitis [2] and the K1 encapsulated strains are most commonly acquired by neonates via vertical transmission from the maternal genital tract in utero or during passage through the birth canal [2]. Most cases of bacterial meningitis in newborns develop as a result of hematogenous spread. One of the most important issues in the pathogenesis of $E$. coli meningitis is how circulating pathogens cross the blood-brain barrier (BBB). Strains causing meningitis possess traits that distinguish them from commensal strains of $E$. coli and other pathogenic strains such as those causing diarrhea and urinary tract infection. Characteristically, meningitic strains of $E$. coli are composed of a restricted number of $\mathrm{O}$ serogroups $(\mathrm{O} 1$, O2, O7, O18, O83), produce S fimbriae, express IbeA, and predominately carry K1 capsule (over 84\%) [1-3].

\footnotetext{
${ }^{*}$ Corresponding author.
}

The presence of these features implies that meningitic strains possess a defined set of virulence determinants that allow the bacterium to penetrate the BBB and enter the central nervous system. An in vitro brain microvascular endothelial cells (BMEC) culture model with characteristics of the BBB has been established for dissecting the molecular and cellular mechanisms of E. coli crossing of the BBB. Using this cell culture model, we and others have shown that efficient penetration of $E$. coli across the BBB is mediated by multiple factors. Several genetic determinants, including $i b e A$, ibeB, yijP, aslA, traJ, cnfI and ompA, are required for $E$. coli crossing of the BBB [1-4]. However, the ibeA gene encoding a $50-\mathrm{kDa}$ protein has been found to be unique to pathogenic $E$. coli $\mathrm{K} 1$ strains (e.g., C5 and RS218), while laboratory strains of E. coli K-12 (e.g., DH5 and HB101) as well as noninvasive $E$. coli $\mathrm{K} 1$ (e.g., E412) lack ibeA [1-3,5-8].

In support of the role of IbeA in E. coli $\mathrm{K} 1$ invasion, bacterial entry of human BMECs involves a complex interplay of IbeA with the host cell receptors and is associated with activation of specific signaling pathways [9-11]. Receptor-mediated mechanisms also play an important 
role in biological functions of nicotine [12]. The involvement of $\alpha 7$ nicotinic acetylcholine receptors $(\alpha 7$ nAChRs) and IbeA-binding proteins (IBPs) in nicotineinduced stimulation of $E$. coli invasion has been demonstrated by using $\alpha 7$ antagonists and IbeA. Soluble IbeA protein markedly abolished both the nicotine-induced enhancement and the baseline activity of bacterial invasion in human BMECs. These findings suggest that IbeA and its receptors play an important role in both the nicotine-induced enhancement and the baseline activity of IbeA $^{+} E$. coli $\mathrm{K} 1$ invasion. These studies suggest that $E$. coli invasion proteins (i.e., IbeA) and their receptors transduce their signal to a common pathway that can be synergistically enhanced by nicotine-mediated signaling by $\alpha 7 \mathrm{nAChRs}$ during $E$. coli invasion of nicotine-stimulated human BMEC. Our studies have further shown that signal transductions and cytoskeletal rearrangements mediated by phosphatidylinositol 3-kinase (PI3K), and calcium signaling-mediated by calmodulin kinase II (Cam$\mathrm{KII})$ are required for $\mathrm{Ibe}^{+} E$. coli $\mathrm{K} 1$ invasion of human BMEC under stimulation with and without nicotine [9-11]. Blocking of PI3K (LY294002) and CamKII (KN93) abolished the entry of $\mathrm{IbeA}^{+}$E. coli $\mathrm{K} 1$ in BMECs in a dose-dependent manner. CamKII-mediated calcium signaling is required for both the IbeA receptor vimentinand $\alpha 7 \mathrm{nAChR}$-induced inflammatory responses [10-11]. These data suggest that the IbeA receptors and $\alpha 7 \mathrm{nAChR}$ could contribute to meningitic inflammation through the common pathway of CamKII-mediated signaling.

In order to understand how $E$. coli invasion proteins interact with BMEC to initiate the signaling required for bacterial entry, a $50-\mathrm{kDa}$ IbeA-binding protein on the surface of human BMECs was identified as vimentin [13]. A similar human BMEC surface protein (IBP55) was previously revealed by immunoblotting with an anti-bovine IBP55 antibody [14]. IbeA, IBP55 and the anti-IBP55 antibody were able to block $E$. coli invasion of human BMEC [14]. However, the identity of IBP55 is unknown. In our further effort to elucidate the role of IbeA-binding proteins in the pathogenesis of E. coli $\mathrm{K} 1$ meningitis, a $100-\mathrm{kDa}$ IbeA-binding protein (IBP100) was purified and identified as polypyrimidine tract-binding protein (PTB)associated splicing factor (PSF) [15]. Protein sequence comparison shows that PSF has significant sequence homology with another RNA-binding protein p54nrb, which is encoded by a different gene. Bladen et al. demonstrated that p54nrb is a pre-mRNA splicing factor [16]. Besides RNA-binding and DNA-binding properties, p54nrb is able to interact with several proteins including PSF and DNA topoisomerase I [15]. In this study we identify IBP55 as p54nrb and demonstrate the role of human p54nrb protein in IbeA-mediated E. coli K1 invasion of human cells. Like another multi-functional intracellular protein nucleolin which is translocated to the Hep-2 cell surface as a receptor for E. coli $\mathrm{O} 157: \mathrm{H} 7$ [17], p54nrb can serve as a binding protein on the BMEC surface for IbeA.

\section{Materials and Methods}

\subsection{Bacterial Strains, Mammalian Cell Lines and Reagents}

E. coli $\mathrm{E} 44$ is a rifampin-resistant virulent strain derived from RS218 (O18:K1:H7) [1,2]. E. coli DH5 $\alpha$ was used for subcloning and BL21 (DE3) for expressing recombinant proteins. The mammalian cells used are human BMEC [2] and human embryonic kidney cell 293T, which were grown at $37^{\circ} \mathrm{C}$ in $5 \% \mathrm{CO}_{2}$ in RPMI1640 medium (Mediatech) with $10 \%$ fetal calf serum. Restriction endonucleases and other enzymes were purchased from New England Biolabs (Beverly, Mass). The anti-IBP55 (Ibe10R) antibody was prepared by immunizing rabbits with bovine BMEC membrane proteins bound onto IbeA-Ni-Sepharose column [14]. Anti-His ${ }_{6}$ and GST monoclonal antibodies were generous gifts from Dr. Yu-Zhou Yang. All other chemicals and antibodies were obtained from Sigma (St. Louis, Mo.) unless otherwise stated.

\subsection{Bioinformatics Approaches}

DNA and protein sequence analysis was done with programs developed by Scientific \& Education Software (Durham, NC). DNA and deduced protein sequences were used to search the DNA and protein databases at NCBI/NIH by using BLAST and PSI-BLAST [18]. SIM alignment program was used for alignment of two protein sequences [6].

\subsection{Protein Expression and Purification}

The expression and purification of recombinant proteins in $E$. coli were carried out according to protocols of manufacturer (Novagen). The GST-IbeA expression construct pGEX17A [15] and the control vector pGEX-KG [19] were transformed into $E$. coli $\mathrm{DH} 5 \alpha$ and the constructs for expression of His tag-containing proteins (pET17A [5], pET28A-p54nrb [20] and pET15b-PSF [21]) were transformed into $E$. coli BL21 (DE3). Purification of GST-IbeA and GST was carried out with glutathione sepharose columns (Pharmacia Biotech). His-tagged proteins purification was performed under denatured condition (8 $\mathrm{M}$ urea) using nickel-nitrilotriacetic acid $\left(\mathrm{Ni}^{2+}\right.$-NTA) agarose columns (Qiagen). The eluted proteins were dialyzed against decreasing concentrations of urea in the same buffer as described previously [5].

\subsection{Isolation of IbeA-Binding Protein with FPLC}

Around $1 \mathrm{ml}$ of $\mathrm{Ni}^{2+}$-NTA agarose (Qiagen) was packed in a HR 5/5 column (Amersham), and washed with 10 
$\mathrm{mM}$ phosphate buffer containing $150 \mathrm{mM} \mathrm{NaCl}$ and $0.2 \%$ Tween 20 at $0.5 \mathrm{ml} / \mathrm{min}$ on the Biologic FPLC system (Bio-Rad). The purified His-IbeA in either active or denatured form was diluted and injected into the column at $0.5 \mathrm{ml} / \mathrm{min}$. After washing for $10 \mathrm{~min}$ with the phosphate buffer, $5 \mathrm{ml}$ of HBMEC crude membrane extract was injected into the IbeA-immobilized column at 0.5 $\mathrm{ml} / \mathrm{min}$. The column was washed extensively, and the binding complex of IbeA was eluted with $250 \mathrm{mM}$ imidazol at $0.5 \mathrm{ml} / \mathrm{min}$ and $1.5 \mathrm{ml}$ of peak was manually collected. The $500 \mu \mathrm{l}$ of the collected sample was precipitated by $10 \%$ TCA and analyzed with SDS-PAGE.

\subsection{SDS-PAGE and Western Blot}

Protein samples were resolved on SDS-12.5\% polyacryamide Phastgels (Amersham) at $16^{\circ} \mathrm{C}$. For Western blot the resolved proteins were transferred onto polyvinylidene fluoride (PVDF) membranes (Millipore). The PVDF membrane was blocked with 5\% non-fat milk powder in PBS plus $0.05 \%$ Tween 20 (PBS-T) at room temperature for $1 \mathrm{~h}$. Then the membrane was incubated in the primary antibody (1:3000 dilution in PBS-T) for $1.5 \mathrm{~h}$ at room temperature, and washed in PBS-T. The horseradish peroxidase (HRP) conjugated secondary antibody (1:5000 dilution in PBS-T) was incubated with the membrane for $1.5 \mathrm{~h}$ at room temperature. After washing the proteins were detected using Lumi-light western blotting substrate kit (Roche).

\subsection{Ligand Overlay Assays}

The ligand overlay assay was carried out as previously described [22]. Briefly HBMECs were lysed in Laemmli sample buffer by boiling for $5 \mathrm{~min}$. The HBMEC lysate was resolved by SDS-PAGE and transferred onto a PVDF membrane. After blocking with 5\% non-fat milk powder the membrane was incubated with $10 \mu \mathrm{g} / \mathrm{ml}$ of GST-IbeA or GST in PBS overnight at $4^{\circ} \mathrm{C}$. Following extensive washing, the GST-IbeA or GST binding spots on the membrane were detected with anti-GST monoclonal antibody as described above.

\subsection{Transfection and Invasion Assays}

The $293 \mathrm{~T}$ of approximately 70\% confluency was transfected with GFP-p54nrb construct pEGFP-C1-p54nrb or GFP construct pEGFP-C1 using the calcium phosphate transfection protocol [23]. The cells were examined under fluorescence microscope after $24 \mathrm{~h}$ incubation. The transfected cells were used for $E$. coli $\mathrm{K} 1$ invasion assays and Western blot. Invasion assays were performed in the cultured HBMEC and $293 \mathrm{~T}$ cells by the method described previously $[1,2]$.

\subsection{Flow Cytometry for the Detection of Cell Surface p54nrb}

Transfected cells were grown to approximately $80 \%$ confluency and harvested with trypsin. Cells were then washed thrice in PBS containing $0.5 \%$ bovine serum albumin (BSA) and incubated in PBS containing 5\% BSA for 30 min. Approximately $3 \times 10^{5}$ cells per sample were incubated with anti-p54nrb monoclonal antibody for 1 hour at room temperature. Following a wash with PBS/BSA solution as described above, cells were incubated with PE-conjugated goat anti-mouse antibody for 1 hour at room temperature in the dark. Again, cells were washed in PBS/FBS and resuspended in PBS/FBS for flow cytometry analysis using a FACSCalibur (BD Biosciences, San Jose, CA). Cells treated with secondary antibody alone served as a negative control for each cell line. A minimum of 10,000 events were scored for each cell line.

\section{Results}

\section{1. p54nrb Shows Significant Sequence Homology with IBP100 Protein (PSF)}

DNA and protein sequences of IBP100 were used to search the DNA and protein databases at the National Center for Biotechnology Information (National Institutes of Health, National Library of medicine, Washington, D.C.) by using the BLAST algorithm. The results indicate that the major portion of PSF protein (241 to 707 residues) shows $59 \%$ identity with almost the entire protein sequence of p54nrb (16 to 471 residues) (Figure 1). Human p54nrb shows a high degree homology with the homologous proteins of rats (96\%) and mouse (97\%) (data not shown), suggesting that p54nrb is a highly conserved protein. However, no homology was found with any other recognized invasion protein receptors, suggesting that PSF and p54nrb represents novel IbeA-binding proteins contributing to E. coli $\mathrm{K} 1$ invasion of BMEC.

\subsection{Interaction between $p 54 n r b$ and IbeA}

PSF has been demonstrated to interact with IbeA and contribute to meningitic E. coli $\mathrm{K} 1$ invasion in vitro [15]. As p54nrb shows significant homology to PSF and also forms a complex with PSF, it might be involved in E. coli $\mathrm{K} 1$ invasion during the development of meningitis. In our previous studies, a polyclonal antibody against bovine IBP55 was generated [14]. To examine whether the antiIBP55 antibody can recognize both PSF and p54nrb, the purified His-PSF and His-p54nrb were blotted with antiIBP55 antibody (Figure 2a). The anti-IBP55 antibody was unable to interact with His-PSF. However, His-p54nrb was clearly recognized by the anti-IBP55 antibody. The recognition of p54nrb was further confirmed by blotting GFP-p54nrb with anti-IBP55 antibody (data not shown). To investigate whether IbeA is able to bind both p54nrb 


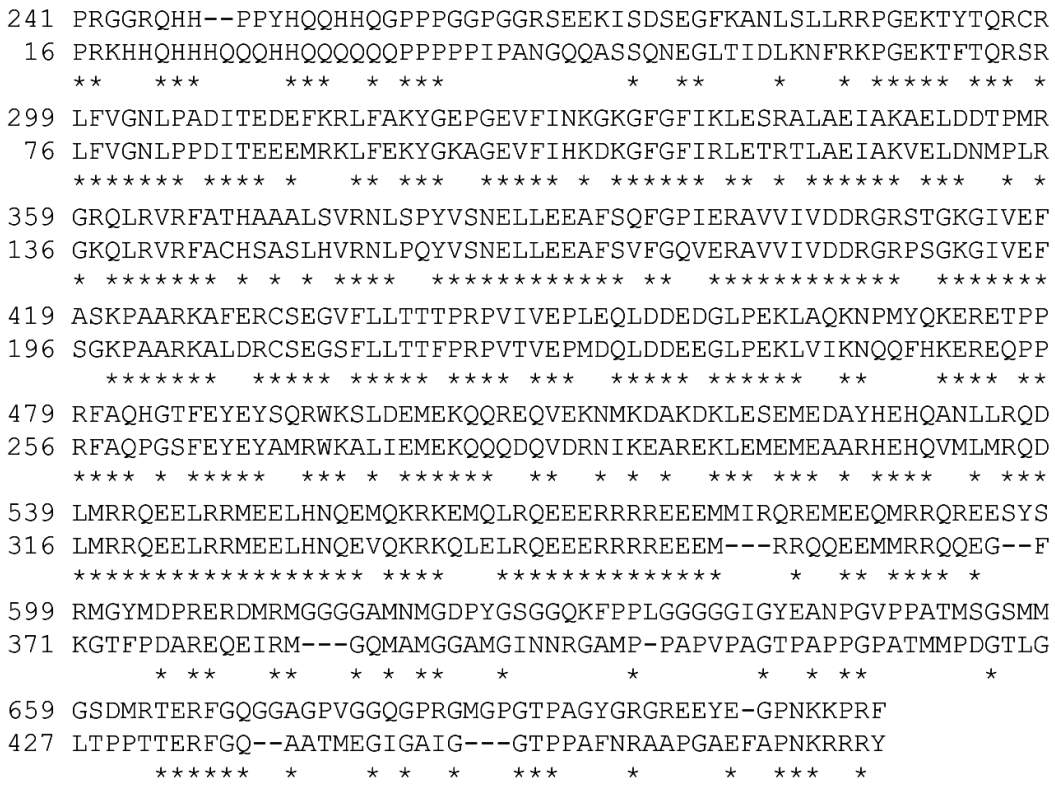

Figure 1. Protein sequence comparison of human IBP100 with IBP55. Amino acid sequences are denoted in single-letter code. The symbol * indicates the identical amino acids.

and PSF, we employed an overlay of Human BMEC lysate with purified GST-IbeA (Figure 2b). Two major bands $(55-\mathrm{kDa}$ and $100-\mathrm{kDa})$ were observed in the GST-IbeA overlay. In contrast, no distinguish bands appeared in the GST overlay control. The positions of $55-\mathrm{kDa}$ and $100-\mathrm{kDa}$ protein bands correspond to the IbeA-binding proteins p54nrb and PSF, respectively. This suggests that IbeA specifically interacts with both p54nrb and PSF.

To further examine the interaction between IbeA and p54nrb, we employed ligand affinity purification. The HBMEC lysate was incubated with Ni-NTA-agarose attached active IbeA or Ni-NTA-agarose alone in eppendorf tubes. After extensive washing, the IbeA-binding proteins were eluted, and p54nrb in the eluates was detected with anti-p54 monoclonal antibody on a western blot (Figure 2c). In contrast to the Ni-NTA-agarose, the eluate from IbeA attached Ni-NTA-agarose contains much stronger signal of p54nrb. To improve the results of affinity purification, we performed the large-scale purification processes on the Biologic FPLC workstation. The HBMEC membrane crude extract was applied onto active IbeA or denatured IbeA saturated Ni-NTA agarose columns. After completion of washing, the IbeA-binding proteins were eluted and analyzed on western blots (Figure 2d). The active IbeA was able to capture p54nrb from crude HBMEC membrane extract. However, no binding of $\mathrm{p} 54 \mathrm{nrb}$ from the crude extract to the column bound with denatured His-tagged IbeA was observed.

\subsection{Soluble p54nrb Competitively Inhibits E. coli K1 Invasion of Human BMEC}

To gain further insight into the relevance of p54nrb in the
IbeA-mediated entry process, we examined the ability of soluble p54nrb to compete with the human BMEC surface receptor for binding of $\mathrm{IbeA}^{+} E$. coli $\mathrm{K} 1$ during the process of bacterial entry. As shown in Figure 3, p54nrb blocked $E$. coli invasion in a dose-dependent manner with a $50 \%$ inhibition at $2.5 \mu \mathrm{g} /$ well $(\mathrm{P}<0.01)$ and an $80 \%$ inhibition at $10 \mu \mathrm{g} /$ well $(\mathrm{P}<0.001)$. In contrast, BSA did not show significant effect on $E$. coli $\mathrm{K} 1$ invasion. We further verified whether p54nrb exhibited any bactericidal effect by incubating the $E$. coli with $54 \mathrm{nrb}$, followed by colony plating. No bactericidal activity was observed (data not shown). These results suggest that the soluble p54nrb is able to prevent bacterial binding to the cell surface of human BMEC, thus blocking E. coli $\mathrm{K} 1$ entry.

\subsection{Overexpressed p54nrb in Mammalian Cells Enhances E. coli K1 Invasion}

To investigate whether overexpression of human p54nrb in $293 \mathrm{~T}$ cells would affect binding and entry of $\mathrm{IbeA}^{+}$E. coli $\mathrm{K} 1$, we transfected $293 \mathrm{~T}$ cells with a plasmid expressing a green fluorescent protein (GFP)-tagged human p54nrb. The GFP intrinsic fluorescence allows direct visualization of transfected cells (Figures $\mathbf{4 a}$ and $\mathbf{4 b}$ ). Transfected cells were tested for their ability to promote binding and entry of $\mathrm{IbeA}^{+} E$. coli $\mathrm{K} 1$. As shown in Figure 4c, IbeA ${ }^{+} E$. coli $\mathrm{K} 1$ invasion was 2 times more efficient in transfected cells than in cells transfected with the vector alone. Western blot was used to verify the GFP-p54nrb expression. The native p54nrb of $54 \mathrm{kDa}$ was detected in both GFP-p54nrb and GFP transfected cells, and the GFP-p54nrb of approximate $80 \mathrm{kDa}$ was only detected in the transfected cells with the GFP-p54nrb plasmid (Figure 4d). As a result of these 

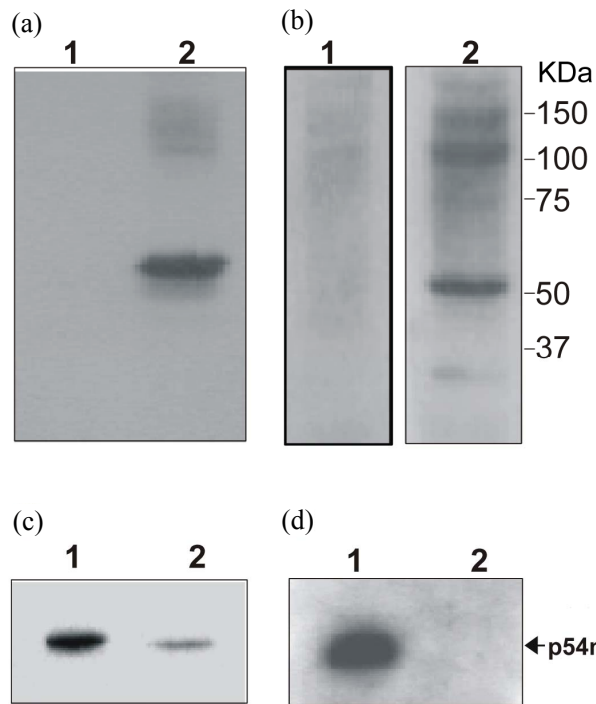

(d)

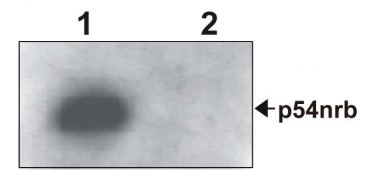

Figure 2. Interaction between p54nrb and IbeA. (a) Anti-IBP55 polyclonal antibody recognizes His-p54nrb. Equal amount of purified His-PSF (Lane 1) and His-p54nrb (Lane 2) were analyzed by Western blot with anti-IBP55 antibody. (b) Overlay of HBMEC with GST-IbeA. The HBMEC lysate was resolved on a SDS-12.5\% polyacryamide gel and transferred on a PVDF membrane. The membrane was blocked with $5 \%$ non-fat milk powder, overlayed with purified GST (Lane 1) or GST-IbeA (Lane 2), and subsequently detected with anti-GST monoclonal antibody. (c), (d) Ligand affinity purification of native p54nrb from HBMEC. (c) The HBMEC lysate was incubated with Ni-NTA-agarose attached His-IbeA (Lane 1) or Ni-NTA-agarose only (Lane 2). After wash with $20 \mathrm{mM}$ imidazole, the protein complex were eluted with $250 \mathrm{mM}$ imidazole and analyzed with anti-p54nrb antibody on a Western blot. (d) The proteins in HBMEC crude membrane extract were allowed to bind to the Ni-NTA-agarose column in the Biologic FPLC system as described in Materials and Methods. The eluates from the columns loaded with active His-IbeA (Lane 1) or denatured His-IbeA (Lane 2) were detected with anti-p54nrb antibody on Western blots. Numbers on the right represents protein molecular mass in $\mathrm{kDa}$.

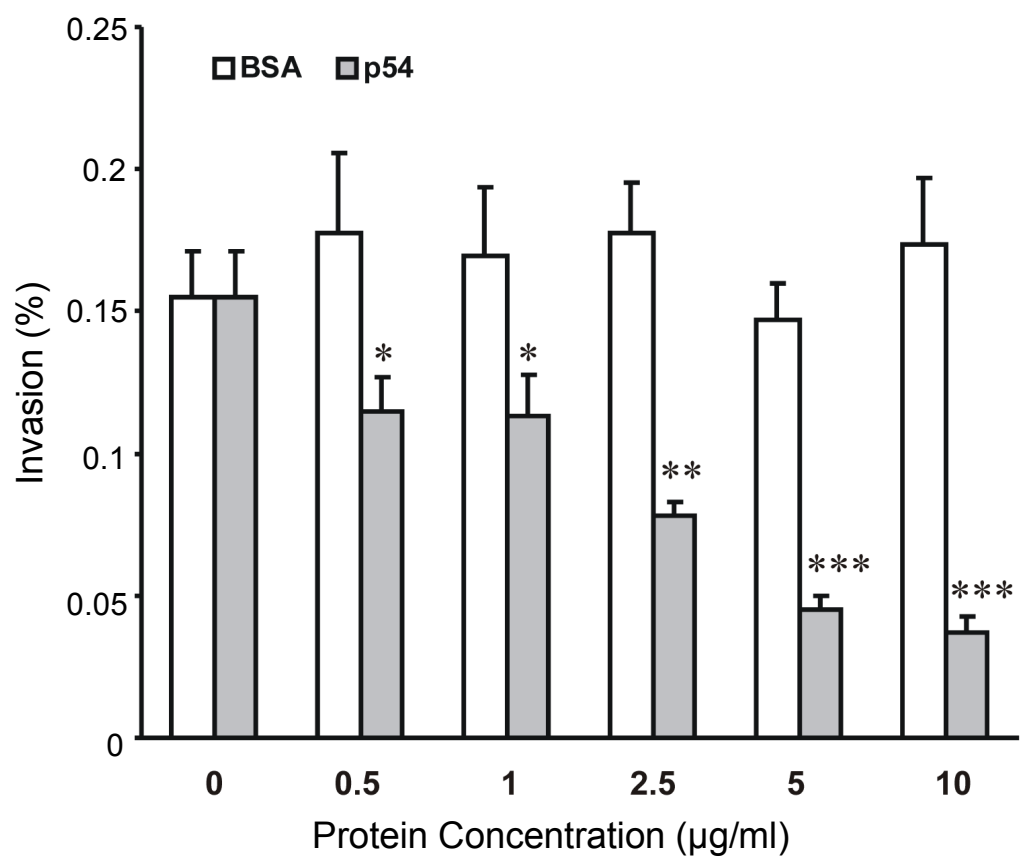

Figure 3. Inhibition of $E$. coli $\mathrm{K} 1$ invasion of HBMEC by His-p54nrb. E44 was incubated with various concentrations of His-p54nrb or BSA for $1 \mathrm{~h}$ on ice before being added to confluent HBMEC monolayers for invasion assays as described in Materials and Methods. Invasion is expressed as percentage of intracellular bacteria among input bacteria. Each value represents the mean of triplicate measurements, and the error bars indicate the standard deviations. The asterisks indicate significantly different from basal levels $(*, * *$ and $* * *$ denote $\mathbf{P}<0.05, \mathbf{P}<0.01$ and $\mathbf{P}<0.001$, respectively). $\mathrm{P}$ values are determined by two-tailed t-test. 
(a)

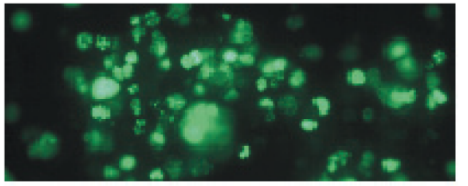

(b)

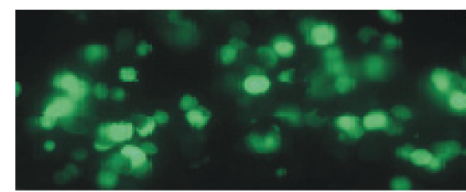

(c)

(d)
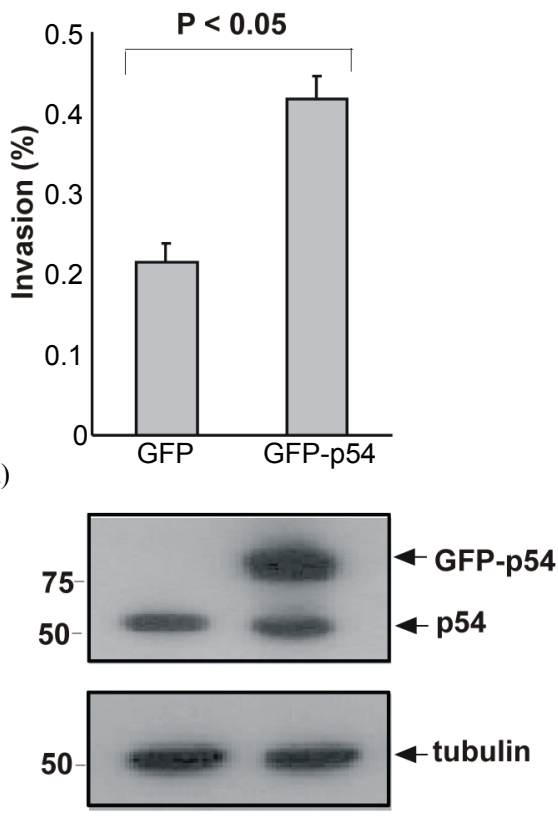

I

II

Figure 4. Enhanced bacterial entry into p54nrb-transfected $293 \mathrm{~T}$ cells. The $293 \mathrm{~T}$ cells were transiently transfected with a plasmid carrying a GFP-p54nrb fusion gene (a) or with a control plasmid carrying a GFP gene (b) are examined by fluorescence microscopy. The invasion assay (c) of the transfectants with GFP-p54nrb or GFP was carried out as described in Materials and Methods. The data represent the mean of triplicate experiments, and the error bars indicate the standard deviations. $P$ value is determined by two-tailed t-test. (d) The transfected 293 T cells with GFP (I) or GFP-p54nrb (II) were lysed and resolved on duplicate SDS-12.5\% PAGE gels. The proteins were transferred onto PVDF membranes and probed with anti-p54nrb or anti-tubulin monoclonal antibody. Numbers on the left represents protein molecular mass in kDa.

findings, we determined the levels of p54nrb expression on the surface of the transfected cells using flow cytometry. As shown in Figure 5, the GFP-p54nrb transfectants have a significantly higher percentage of cells expressing cell surface p54nrb compared to the GFP transfectants. These results further supported that enhanced E. coli K1 invasion of the GFP-p54nrb transfectants resulted from over-expression of $\mathrm{p} 54 \mathrm{nrb}$. Taken together, the transfection experiments indicate that human p54nrb plays a critical role in the internalization step.

\section{Discussions}

The fundamental issue in the molecular pathogenesis of $E$. coli $\mathrm{K} 1$ meningitis is how specific surface structures on both microbial pathogens and host cells contribute to the tissue tropism of this disease. There are several $E$. coli $\mathrm{K} 1$ genetic determinants and host cell surface proteins contributing to bacterial invasion of human BMEC [1-7]. The bacterial factors include IbeA, IbeB, YijP, AslA, OmpA, TraJ and CNF1 [2-4]. Among these invasion determinants, the ibeA gene is significantly associated with meningitic $E$. coli $\mathrm{K} 1$ strains isolated from CSF in comparison with $E$. coli strains from intestinal and vaginal microbiota, amniotic fluid and blood $[8,24]$. The host cell surface binding proteins or receptors present on human BMEC include vimentin and PSF for IbeA [13,15], Ecgp96 for OmpA [25] and laminin receptor for CNF1 [26]. However, it is not clear whether and how the PSF partner protein $\mathrm{p} 54 \mathrm{nrb}$ contributes to $E$. coli $\mathrm{K} 1$ invasion of BMEC. In our study to further elucidate the role of IbeA-binding proteins in E. coli $\mathrm{K} 1$ invasion of human BMEC, integrating affinity chromatography and Biacore analysis with mass spectrometry has allowed us to detect and characterize molecular interactions between IbeA and its partners to be complemented with identifycation of a $100-\mathrm{kDa}$ IbeA-binding protein as PSF. This protein shows significant sequence homology with another RNA-binding protein p54nrb [16]. It is unlikely that this protein is a degraded product of PSF since they are encoded by two different genes [16,20,21]. The specific interaction between IbeA and p54nrb has been further demonstrated by several independent approaches. These include 1) a specific reaction of $\mathrm{p} 54 \mathrm{nrb}$ with the an anti-IBP55 antibody; 2) the ability of p54nrb to bind to IbeA with ligand overlay assays and ligand affinity purification; 3) inhibition of $E$. coli invasion by soluble p54nrb; 4) detection of increased p54nrb expression on the cell surface of the GFP-p54nrb transfectants by flow cytometry; and 5) overexpression of p54nrb in the transfectants resulting in the increased invasion of $E$. coli $\mathrm{K} 1$. 


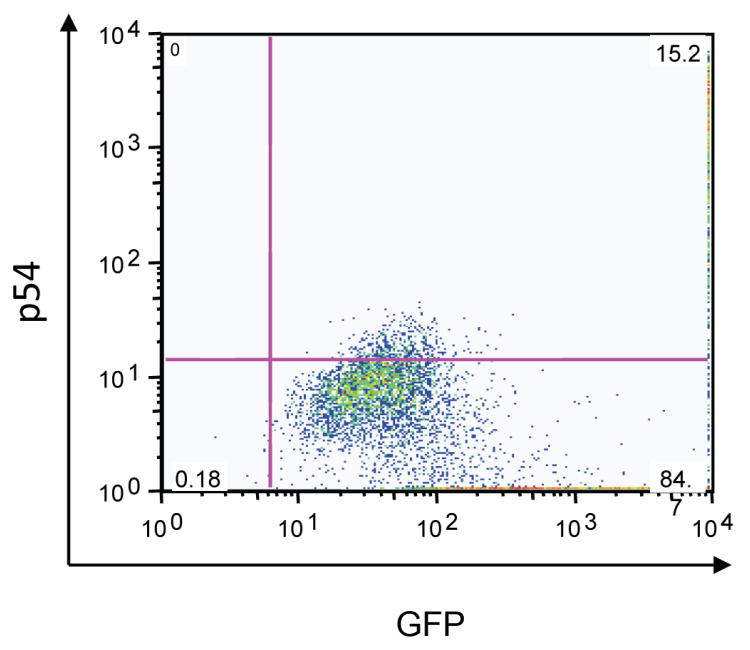

(a)

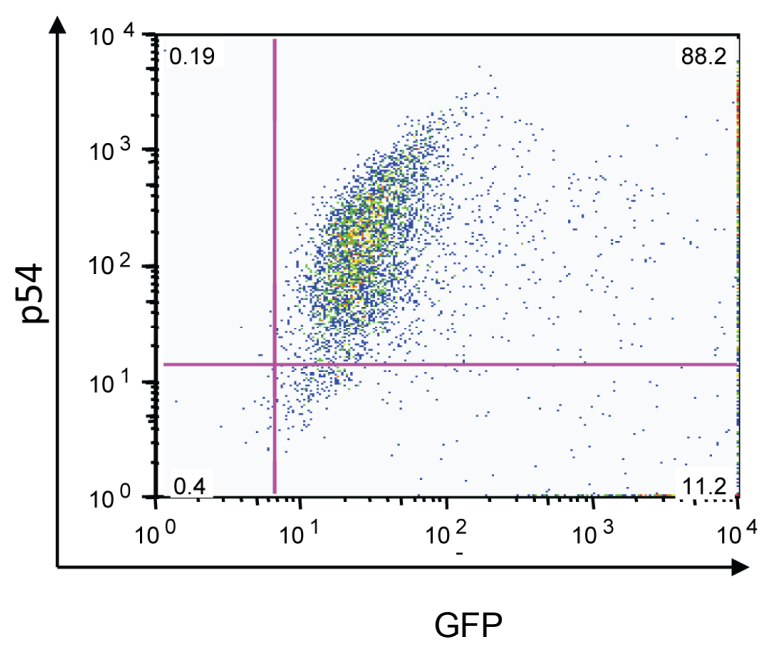

(b)

Figure 5. Analysis of surface p54nrb expression in the transfectants using flow cytometry. 293T cells were transiently transfected with a control plasmid carrying a GFP gene (a) or with a plasmid carrying a GFP-p54nrb fusion gene (b). Cell surface p54nrb expression was determined using immunofluorescence of anti-p54nrb antibody plus secondary antibody. Percentages represent the percent population of cells expressing p54nrb. The GFP-p54nrb transfectants had a significantly higher percentage of cells expressing p54nrb than the GFP transfectants.

p54nrb represents an abundant and ubiquitous class of protein with 471 amino acid residues. Originally, p54nrb was purified as a heterodimer with PSF, which shows extensive sequence homology to p54nrb [27]. Copurification of p54nrb and PSF has been observed in a number of studies $[16,20,27]$. PSF and p54nrb are steroid receptor corepressors [28]. Both proteins contain protein phosphatase 1 (PP1) consensus binding RVxF motifs, suggesting that PP1 may regulate the phosphorylation status of p54nrb and PSF and thus their function in gene transcription. A recent study demonstrated that PP1 could directly interact with the RVxF motif only in p $54 \mathrm{nrb}$, but not in PSF [28]. p54nrb has been shown to be implicated in numerous cellular processes including transcriptional regulation, splicing, DNA unwinding, nuclear retention of hyperedited double-stranded RNA, viral RNA processing, control of cell proliferation, and circadian rhythm maintenance $[16,20,21,27]$. It can bind single and doublestranded RNA and DNA and has inherent carbonic anhydrase activity. A number of reports suggest that p54nrb participates in coordinated regulation of both cell surface and nuclear receptors, including receptors for IL-17 [29] and steroid hormones, estrogen and androgen [30,31]. It has been suggested that p54nrb mediates different functions depending on its intracellular location, which could be regulated by phosphorylation. This protein can be phosphorylated on multiple sites including tyrosine, serine and threonine residues [32]. Our studies demonstrated that p54nrb could act as a cell surface protein contributing to E. coli K1 entry into human BMEC. Similarly, a number of intracellular proteins such as OmpA-binding protein (Ecgp96), glucose transporters, T-plastin and calnexin have been shown to translocate from the intracellular compartments to the plasma membrane [25,33-35]. Another multi-functional protein nucleolin, which is localized predominantly in the nucleolus, has been proved to be the Hep-2 cell surface receptor for an outer membrane protein intimin- $\gamma$ of $E$. coli $\mathrm{O} 157: \mathrm{H} 7$ [17]. Nucleolin is an RNA-binding protein and also an autoantigen. Nucleolin contains three major domains through which involve the organization of nucleolar chromatin, packaging of preRNA, rDNA transcription, and ribosome assembly [36]. Like nucleolin and PSF, p54nrb is a multi-functional regulatory protein located in both nucleolus and cell surface. This phenomenon may represent a mechanism for signal transduction between the nuclear compartment and the cell surface [36].

Our studies showed that overexpression of $\mathrm{p} 54 \mathrm{nrb}$ in 293Tcells (normally noninvasive for $E$. coli K1) make these cells more susceptible to $E$. coli $\mathrm{K} 1$ invasion, suggesting that $\mathrm{p} 54 \mathrm{nrb}$ indeed is responsible for $\mathrm{IbeA}^{+} E$. coli $\mathrm{K} 1$ invasion of human BMEC. Despite the accumulation of data suggesting that $i b e A$ plays a crucial role in E. coli $\mathrm{K} 1$ invasion of human BMEC, a coordinated reaction of IbeA with other invasion proteins, such as IbeB, AslA, YijP, OmpA and CNF1, may be required for the entire invasion process. It has been demonstrated that OmpA- and CNF1-induced invasion are mediated by human BMEC receptors $[25,26]$. Since IbeA is unique to meningitic E. coli $\mathrm{K} 1$, the interaction of IbeA with human BMEC via PSF and p54nrb appears to be more crucial for the invasion process. Like PSF, p54nrb has been demonstrated to exhibit multiple functions include binding of nucleic acids (DNA and RNA) and proteins, DNA 
pairing, promotion of pre-mRNA splicing, and transcripttional regulation [16,20,21]. Due to its dual RNA/ DNA-binding properties and the ability to interact with several protein counterparts, $\mathrm{p} 54 \mathrm{nrb}$ might be regarded as a "sticky" protein that can link multiple gene regulation processes, which may play a crucial role in the $E$. coli $\mathrm{K} 1$ invasion process. Recently, we have demonstrated that vimentin and PSF could cooperatively contribute to $\mathrm{IbeA}^{+}$ $E$. coli $\mathrm{K} 1$-induced $\mathrm{NF}-\kappa \mathrm{B}$ signaling, bacterial invasion and leukocyte transmigration across the BBB [37], which are three hallmark features of bacterial meningitis [38]. Thus, it is tempting to speculate that IbeA/vimentin/ $\mathrm{PSF} / \mathrm{p} 54 \mathrm{nrb}$ interactions may represent an important mechanism responsible for a coordinated modulation of both bacterial and host factors that contribute to the invasion process.

In summary, the data presented in this report establish that $\mathrm{p} 54 \mathrm{nrb}$ is the $55-\mathrm{kDa}$ IbeA-binding protein, IBP55, which mediates E. coli K1 invasion of BMEC. Further work is needed to examine how vimentin, PSF and p54nrb coordinately contribute to IbeA-induced signal transduction and subsequently E. coli $\mathrm{K} 1$ entry into the host cells.

\section{Acknowledgements}

We thank Dr. Kwang S Kim for providing brain endothelial cells, Dr. Prasadarao V. Nemani for the polyclonal antibody against IBP55, Dr. Yanming Zou for Western blot and ligand overlay assays, Dr. Zhi-Hua Xie (ProteinX Lab) for protein purification, Dr. Yu-Zhou Yang for anti-His and anti-GST monoclonal antibodies, and Dr. James G. Patton for p54nrb and PSF constructs. This project is financially supported by Public Health Service grants R01-AI40635 (S.H.H.) and R01-NS047599 (A.J.), and China Natural Science Foundation grant 81070514 (L.W).

\section{REFERENCES}

[1] S. H. Huang, C. A. Wass, Q. Fu, N. V. Prasadarao, M. F. Stins and K. S. Kim, "Escherichia coli Invasion of Brain Microvascular Endothelial Cells in Vitro and in Vivo: Molecular Cloning and Characterization of Invasion Gene ibe 10," Infection and Immunity, Vol. 63, No. 11, 1995, pp. 4470- 4475.

[2] S. H. Huang, M. Stins and K. S. Kim, "Bacterial Penetration across the Blood-Brain Barrier during the Development of Neonatal Meningitis," Microbes and Infection, Vol. 2, No. 10, 2000, pp. 1237-1244. doi:10.1016/S1286-4579(00)01277-6

[3] S. H. Huang and A. Jong, "Cellular Mechanisms of Microbial Proteins Contributing to Invasion of the Blood-Brain Barrier," Cellular Microbiology, Vol. 3, No. 5, 2001, pp. 277-287. doi:10.1046/j.1462-5822.2001.00116.x

[4] K. S. Kim, "Pathogenesis of Bacterial Meningitis: From
Bacteraemia to Neuronal Injury," Nature Reviews Neuroscience, Vol. 4, No. 5, 2003, pp. 376-385.

doi:10.1038/nrn1103

[5] S. H. Huang, Z. S. Wan, Y. H. Chen, A. Y. Jong and K. S. Kim, "Further Characterization of Escherichia coli Brain Microvascular Endothelial Cell Invasion Gene ibeA by Deletion, Complementation, and Protein Expression," Journal of Infectious Diseases, Vol. 183, No. 7, 2001, pp. 1071-1078. doi:10.1086/319290

[6] S. H. Huang, Y. H. Chen, G. Y. Kong, S. H. Chen, M. Borodovsky, J. Besemer and A. Y. Jong, "A Novel Genetic Island of Meningitic E. coli $\mathrm{K} 1$ Containing the ibeA Invasion Gene (GimA): Functional Annotation and Carbon Source-Regulated Invasion of Human Endothelial Cells," Functional \& Integrative Genomics, Vol. 1, No. 5, 2001, pp. 312-322. doi:10.1007/s101420100039

[7] S. H. Huang, P. Germon and A. Y. Jong, "The InvasionAssociated Genetic Island GimA in Meningitic E. coli K1," In: F. Columbus, Ed., Focus on Meningitis Research, Nova Science Publisher, New York, 2004, pp. 97-114.

[8] S. Bonacorsi, O. Clermont, V. Houdouin, C. Cordevant, N. Brahimi, A. Marecat, C. Tinsley, X. Nassif, M. Lange and E. Bingen, "Molecular Analysis and Experimental Virulence of French and North American Escherichia coli Neonatal Meningitis Isolates: Identification of a New Virulent Clone," Journal of Infectious Diseases, Vol. 188, No. 7, 2003, pp. 1895-1906. doi:10.1086/375347

[9] Y. H. Chen, S. H. Chen, A. Jong, Z. Y. Zhou, W. Li, K. Suzuki and S. H. Huang, "Enhanced Escherichia coli Invasion of Human Brain Microvascular Endothelial Cells Is Associated with Alterations in Cytoskeleton Induced by Nicotine," Cellular Microbiology, Vol. 4, No. 8, 2002, pp. 503-514. doi:10.1046/j.1462-5822.2002.00209.x

[10] F. Chi, T. Jong, L. Wang, Y. Ouyang, C. Wu, W. Li and S. H. Huang, "Vimentin-Mediated Signaling Is Required for Ibe $\mathrm{A}^{+} E$. coli $\mathrm{K} 1$ Invasion of Human Brain Microvascular Endothelial Cells," Biochemical Journal, Vol. 427, No. 1, 2010, pp. 79-90. doi:10.1042/BJ20091097

[11] F. Chi, L. Wang, X. Zheng, A. Jong and S. H. Huang, "Recruitment of $\alpha 7$ Nicotinic Acetylcholine Receptor to Caveolin-1-enriched Lipid Rafts is Required for Nicotine-Enhanced Escherichia coli K1 Entry into Brain Endothelial Cells," Future Microbiology, Vol. 6, No. 8, 2011, pp. 953-966.

[12] T. Kihara, S. Shimohama, H. Sawada, K. Honda, T. Nakamizo and H. Shibasaki, "Alpha 7 Nicotinic Receptor Transduces Signals to Phosphatidylinositol 3-Kinase to Block A Beta-Amyloid-Induced Neurotoxicity," Journal of Biological Chemistry, Vol. 276, No. 17, 2001, pp. 13541-13546.

[13] Y. Zou, L. He and S. H. Huang, "Identification of a Surface Protein on Human Brain Microvascular Endothelial Cells as Vimentin Interacting with Escherichia coli Invasion Protein IbeA," Biochemical and Biophysical Research Communications, Vol. 351, No. 3, 2006, pp. 625-630. doi:10.1016/j.bbrc.2006.10.091

[14] N. V. Prasadarao, C. A. Wass, S. H. Huang and K. S. Kim, "Identification and Characterization of a Novel Ibe10 Binding Protein that Contributes to Escherichia coli In- 
vasion of Brain Microvascular Endothelial Cells," Infection and Immunity, Vol. 67, No. 3, 1999, pp. 1131-1138.

[15] Y. Zou, L. He, C. H. Wu, H. Cao, Z. H. Xie, Y. Wang, A. Y. Jong and S. H. Huang, "PSF Is an IbeA-Binding Protein Contributing to Meningitic Escherichia coli K1 Invasion of Human Brain Microvascular Endothelial Cells," Medical Microbiology and Immunology, Vol. 196, No. 3, 2007, pp. 135-143. doi:10.1007/s00430-006-0034-x

[16] C. L. Bladen, D. Udayakumar, Y. Takeda and W. S. Dynan, "Identification of the Polypyrimidine Tract Binding Protein-Associated Splicing Factor p54(nrb) Complex as a Candidate DNA Double-Strand Break Rejoining Factor," Journal of Biological Chemistry, Vol. 280, No. 7, 2005, pp. 5205-5210. doi:10.1074/jbc.M412758200

[17] J. F. Sinclair and A. D. O'Brien, "Cell Surface-Localized Nucleolin Is a Eukaryotic Receptor for the Adhesin Intimin-Gamma of Enterohemorrhagic Escherichia coli O157:H7," Journal of Biological Chemistry, Vol. 277, No. 4, 2002, pp. 2876-2885. doi:10.1074/jbc.M110230200

[18] S. F. Altschul, T. L. Madden, A. A. Schäffer, J. Zhang, Z. Zhang, W. Miller and D. J. Lipman, "Gapped BLAST and PSI-BLAST: A New Generation of Protein Database Search Programs," Nucleic Acids Research, Vol. 25, No. 7, 1997, pp. 3389-3402. doi:10.1093/nar/25.17.3389

[19] K. Guan and J. E. Dixon, "Eukaryotic Proteins Expressed in Escherichia coli: An Improved Thrombin Cleavage and Purification Procedure of Fusion Proteins with Glutathione S-Transferase," Analytical Biochemistry, Vol. 192, No. 2, 1991, pp. 262-267. doi:10.1016/0003-2697(91)90534-Z

[20] R. Peng, B. T. Dye, I. Perez, D. C. Barnard, A. B. Thompson and J. G. Patton, "PSF and p54nrb Bind a Conserved Stem in U5 snRNA," RNA, Vol. 8, No. 10, 2002, pp. 1334-1347. doi:10.1017/S1355838202022070

[21] B. T. Dye and J. G. Patton, "An RNA Recognition Motif (RRM) Is Required for the Localization of PTB Associated Splicing Factor (PSF) to Subnuclear Speckles," Experimental Cell Research, Vol. 263, No. 1, 2001, pp. 131144. doi:10.1006/excr.2000.5097

[22] L. Braun, B. Ghebrehiwet and P. Cossart, "gC1q-R/p32, a C1q-Binding Protein, Is a Receptor for the InIB Invasion Protein of Listeria monocytogenes," EMBO Journal, Vol. 19, No. 7, 2000, pp. 1458-1466. doi:10.1093/emboj/19.7.1458

[23] R. E. Kingston, C. A. Chen and H. Okayama, "Calcium Phosphate Transfection," In: J. S. Bonifacino, M. Dasso, J. B. Harford, J. L. Schwartz and K. M. Yamada, Eds., Current Protocols in Cell Biology, John Wiley \& Sons, New York, 2003, pp. 2031-2038. doi:10.1002/0471143030.cb2003s19

[24] S. Watt, P. Lanotte, L. Mereghetti, M. M. Schouleur, B. Picard and R. Quentin, "Escherichia coli Strains from Pregnant Women and Neonates: Intraspecies Genetic Distribution and Prevalence of Virulence Factors," Journal of Clinical Microbiology, Vol. 41, No. 5, 2003, pp. 19291935. doi:10.1128/JCM.41.5.1929-1935.2003

[25] N. V. Prasadarao, P. K. Srivastava, R. S. Rudrabhatla, K. S. Kim, S. H. Huang and S. K. Sukumaran, "Cloning and Expression of the Escherichia coli K1 Outer Membrane
Protein A Receptor, a gp96 Homologue," Infection and Immunity, Vol. 71, No. 4, 2003, pp. 1680-1688. doi:10.1128/IAI.71.4.1680-1688.2003

[26] K. J. Kim, J. W. Chung and K. S. Kim, “67-kDa Laminin Receptor Promotes Internalization of Cytotoxic Necrotizing Factor 1-Expressing Escherichia coli K1 into Human Brain Microvascular Endothelial Cells," Journal of Biological Chemistry, Vol. 280, No. 2, 2005, pp. 1360-1368. doi:10.1074/jbc.M410176200

[27] A. H. Fox, C. S. Bond and A. I. Lamond, "P54nrb Forms a Heterodimer with PSP1 That Localizes to Paraspeckles in an RNA-Dependent Manner," Molecular Biology of the Cell, Vol. 16, No. 11, 2005, pp. 5304-5315.

doi:10.1091/mbc.E05-06-0587

[28] L. Liu, N. Xie, P. Rennie, J. R. Challis, M. Gleave, S. J. Lye and X. Dong, "Consensus PP1 Binding Motifs Regulate Transcriptional Corepression and Alternative RNA Splicing Activities of the Steroid Receptor Coregulators, p54nrb and PSF," Molecular Endocrinology, Vol. 25, No. 7, 2011, pp. 1197-1210. doi:10.1210/me.2010-0517

[29] K. Thiele, D. Riemann, S. A. Navarrete, J. Langner and A Kehlen, "Cell-Cell Contact of Human T Cells with Fibroblasts Changes Lymphocytic mRNA Expression: Increased mRNA Expression of Interleukin-17 and Interleukin-17 Receptor," European Cytokine Network, Vol. 11, No. 1, 2000, pp. 53-58.

[30] K. Ishitani, T. Yoshida, H. Kitagawa, H. Ohta, S. Nozawa and S. Kato, "p54nrb Acts as a Transcriptional Coactivator for Activation Function 1 of the Human Androgen Receptor," Biochemical and Biophysical Research Communications, Vol. 306, No. 3, 2003, pp. 660-665. doi:10.1016/S0006-291X(03)01021-0

[31] O. Adegbola and G. R. Pasternack, “A pp32-Retinoblastoma Protein Complex Modulates Androgen Receptor-Mediated Transcription and Associates with Components of the Splicing Machinery," Biochemical and Biophysical Research Communications, Vol. 334, No. 2, 2005, pp. 702-708. doi:10.1016/j.bbrc.2005.06.153

[32] A. Proteau, S. Blier, A. L. Albert, S. B. Lavoie, A. M. Traish and M. Vincent, "The Multifunctional Nuclear Protein p54nrb Is Multiphosphorylated in Mitosis and Interacts with the Mitotic Regulator Pin1," Journal of Molecular Biology, Vol. 436, No. 4, 2005, pp. 1163-1172. doi:10.1016/j.jmb.2004.12.034

[33] T. Adam, M. Arpin, M. C. Prevost, P. Gounon and P. J. Sansonetti, "Cytoskeletal Rearrangements and the Functional Role of T-Plastin during Entry of Shigella flexneri into HeLa Cells," Journal of Cell Biology, Vol. 192, No. 2, 1995, pp. 367-381. doi:10.1083/jcb.129.2.367

[34] D. Zhou, M. S. Mooseker and J. E. Galan, “An Invasion-Associated Salmonella Protein Modulates the Actin-Bundling Activity of Plastin," Proceeding of the $\mathrm{Na}$ tional Academy of Sciences of the United States of America, Vol. 96, No. 18, 1999, pp. 10176-10181.

[35] N. Manel, F. J. Kim, S. Kinet, N. Taylor, M. Sitbon and J. L. Battini, "The Ubiquitous Glucose Transporter GLUT-1 Is a Receptor for HTLV," Cell, Vol. 115, No. 4, 2003, pp. 449-459. doi:10.1016/S0092-8674(03)00881-X

[36] M. Srivastava and H. B. Pollard, "Molecular Dissection 
of Nucleolin's Role in Growth and Cell Proliferation: New Insights," FASEB Journal, Vol. 13, No. 14, 1999, pp. 1911-1922.

[37] F. Chi, T. Bo, C. H. Wu, A. Jong and S. H. Huang, "Vimentin and PSF Act in Concert to Regulate IbeA" Escherichia coli K1-Induced Activation and Nuclear Translocation of NF- $\kappa \mathrm{B}$ in Human Brain Microvascular
Endothelial Cells," PLoS One, in Press, 2012.

[38] X. Che, F. Chi, L. Wang, T. Jong, C. H. Wu, X. Wang and S. H. Huang, "Involvement of IbeA in Meningitic Escherichia coli K1-Induced Polymorphonuclear Leukocyte Transmigration across Brain Endothelial Cells," Brain Pathology, Vol. 21, No. 4, 2011, pp. 389-404. doi:10.1111/j.1750-3639.2010.00463.x

\section{Abbreviations}

PSF, polypyrimidine tract-binding protein (PTB)-associated splicing factor; BMEC, brain microvascular endothelial cells; p54nrb, 54-kDa nuclear RNA-binding

protein; CSF, cerebrospinal fluid; BBB, blood-brain barrier; IBP55, 55-kDa IbeA-binding protein; IBP100, $100-\mathrm{kDa}$ IbeA-binding protein. 